\title{
Dislocation dynamics of strain relaxation in epitaxial layers
}

\author{
T. C. Wang ${ }^{\text {a) }}$ \\ LNM, Institute of Mechanics, Chinese Academy of Science, Beijing 100080, People's Republic of China
}

Y. W. Zhang and S. J. Chua

Institute of Materials Research and Engineering, National University of Singapore, Singapore 117602, Singapore

(Received 28 August 2000; accepted for publication 8 March 2001)

\begin{abstract}
Many experimental observations have clearly shown that dislocation interaction plays a crucial role in the kinetics of strain relaxation in epitaxial thin films. A set of evolution equations are presented in this article. The key feature of the equations is the incorporation of dislocation interactions into the kinetic process by introducing a resistance term. The resistance to threading dislocation gliding is characterized by a hardening function, which depends only on the relaxed plastic strain. The evolution equations are tested on the $\mathrm{Ge}_{x} \mathrm{Si}_{1-x} / \mathrm{Si}(100)$ materials system. Existing fundamental parameters are incorporated into the present model. The evolution equations successfully reproduce a wide range of experimental data on strain relaxation in $\mathrm{Ge}_{x} \mathrm{Si}_{1-x} / \mathrm{Si}$ heterostructures. (C) 2001 American Institute of Physics. [DOI: 10.1063/1.1369396]
\end{abstract}

\section{INTRODUCTION}

During the past 25 years a great deal of theoretical and experimental research has focused on strain relaxation by creating mismatch dislocations in lattice mismatched epitaxial thin film. ${ }^{1,2}$ Relaxed epitaxial layers, especially composition graded buffer layers, have recently been created with high quality due to an understanding of the kinetics of epitaxial growth conditions. The major advances include insight into the dislocation dynamics and control of the surface roughness. ${ }^{3,4}$ An early kinetic description of strain relaxation by misfit dislocations was given by Dodson and Tsao. ${ }^{5}$ They proposed a simple phenomenological model to describe the plastic strain rate. Hull et al. ${ }^{6}$ developed a kinetic model to simulate the relaxation process. Houghton ${ }^{7}$ proposed a set of evolution equations to interconnect the nucleation and propagation of threading dislocations and the formation of mismatch dislocations. Subsequent models ${ }^{3,8}$ have been proposed along a similar line. A sound model of the strain relaxation should include the major elements of nucleation, propagation, interaction, and multiplication of dislocations.

In this article we propose a set of evolution equations to describe the essential process for strain relaxation. The key feature of the equations is the incorporation of dislocation interactions into the kinetic process. In order to get a clear picture of the kinetic process of strain relaxation, we will focus our attention on a uniform single epitaxial layer deposited on a coherent thick substrate.

\section{FORMULATIONS}

The relaxed plastic strain rate can be expressed by the following equation: ${ }^{2,9}$

$$
\dot{\epsilon}_{p}=\rho b_{1} v / 2,
$$

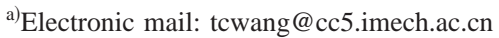

where $\rho$ is the mobile threading dislocation density (the number of mobile threading dislocations per unit area of interface), $b_{1}$ is the threading dislocation component parallel to the interface and perpendicular to corresponding misfit dislocation lines, and $v$ is the mean velocity of the threading dislocation. The evolution equations take the form

$$
\begin{aligned}
& v=v_{0}\left(\frac{\tau_{\mathrm{exc}}-\tau_{R}}{\mu}\right)^{m} \exp \left(-\frac{Q_{v}}{k T}\right), \\
& \dot{\rho}=\xi_{0}\left(\frac{\tau_{\mathrm{exc}}-\tau_{R}}{\mu}\right)^{n} \exp \left(-\frac{Q_{\rho}}{k T}\right),
\end{aligned}
$$

where $\mu$ is the shear modulus of the epitaxial layer, $Q_{v}$ and $Q_{\rho}$ are the activation energies for the velocity and the threading dislocation density, respectively, $k$ is the Boltzmann constant, $T$ is the absolute temperature, and $\tau_{\mathrm{exc}}$ is the excess stress, which can be expressed as ${ }^{1,2}$

$$
\tau_{\mathrm{exc}}=c \mu\left(\left(\epsilon_{0}-\epsilon_{p}\right)-K_{0} \frac{\ln (2 h / b)}{h \ln \left(2 h_{0} / b\right)}\right),
$$

where $\epsilon_{0}$ is the mismatch strain, $\epsilon_{p}$ is the relaxed plastic strain, and $c=2(1+\nu) /(1-\nu)$, where $\nu$ is the Poisson ratio. $v_{0}$ and $\xi_{0}$ are the material parameters.

For a network of $60^{\circ}$ misfit dislocations $K_{0}$ is expressed by

$$
K_{0}=\frac{b(1-\nu / 4)}{4 \pi(1+\nu)} \ln \left(2 h_{0} / b\right),
$$

where $h_{0}$ is a reference thickness and $b$ is the Burgers vector. For the convenience of engineering applications, Tersoff $\mathrm{f}^{10}$ assumed that $K_{0}$ can be taken as a material constant and the variation of the function $\ln (2 h / b) / \ln \left(2 h_{0} / b\right)$ is much weaker than the variation of the function $1 / h$, hence Eq. (4) can be simplified to

$$
\tau_{\mathrm{exc}}=c \mu\left(\left(\epsilon_{0}-\epsilon_{p}\right)-\frac{K_{0}}{h}\right),
$$




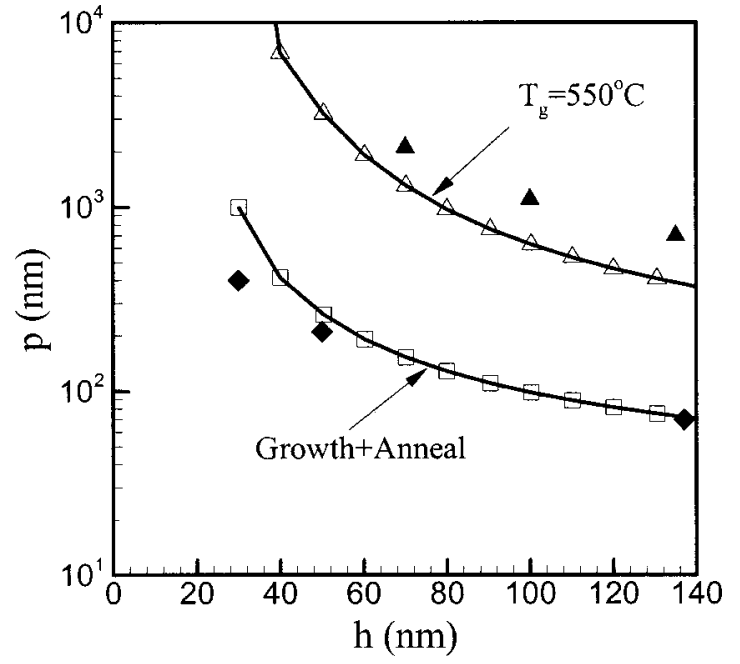

FIG. 1. Comparison of experiment and present modeling for growth and growth annealing of an $x=0.25 \mathrm{Ge}_{x} \mathrm{Si}_{1-x} / \mathrm{Si}(100)$ structure. The experimental data, shown by closed points, were given by Hull et al. (see Ref. 6) for $550{ }^{\circ} \mathrm{C}$ growth and a growth +10 min in situ anneal at $800{ }^{\circ} \mathrm{C}$ in the MBE chamber for the average measured dislocation spacing $p$ vs epitaxial layer thickness $h$. The growth rate is $0.3 \mathrm{~nm} \mathrm{~s}^{-1}$. All open points are the present simulation results.

$\tau_{R}$ is the resistance to the threading dislocation gliding arising from dislocation interaction and surface roughness, and can be expressed as

$$
\tau_{R}=c \mu H\left(\epsilon_{p}\right)=c \mu \alpha\left(\frac{\epsilon_{p}}{\epsilon_{L}}\right)^{\beta}\left[1-\tanh \left(\frac{\gamma \epsilon_{p}}{\epsilon_{L}}\right)\right],
$$

where $\epsilon_{L}$ is the maximum misfit between the epitaxial film and the thick substrate. For the $\mathrm{Ge}_{x} \mathrm{Si}_{1-x} / \mathrm{Si}$ system, $\epsilon_{L}$ $=0.0418($ at $x=1)$. The parameters $\alpha, \beta$, and $\gamma$ are material constants. Usually the function $H\left(\epsilon_{p}\right)$ is called the work hardening function. The power law hardening function $\alpha\left(\epsilon_{p} / \epsilon_{L}\right)^{\beta}$ is well known in plasticity theory. The term [1 $\left.-\tanh \left(\gamma \epsilon_{p} / \epsilon_{L}\right)\right]$ prevents the material behavior from hardening too quickly. When $\gamma=0$, the epitaxial film becomes a pure power law hardening material.

The relaxed plastic strain $\epsilon_{p}$ is directly related to the average mismatch dislocation spacing $p$, according to the following formula:

$$
\epsilon_{p}=b_{1} / p \text {. }
$$

\section{DETERMINATION OF MATERIAL PARAMETERS}

The evolution equations are tested on the $\mathrm{Ge}_{x} \mathrm{Si}_{1-x} / \mathrm{Si}$ material system. The material parameters $m, n$, and $Q_{v}$ and $Q_{\rho}$ were experimentally measured by Hull et al. ${ }^{6}$ and by Houghton. ${ }^{7}$ In this article we use the following data: ${ }^{6,7} \mathrm{~m}$ $=2.0, n=2.5, Q_{v}=1.1 \mathrm{eV}$, and $Q_{\rho}=2.2 \mathrm{eV}$. The materials parameters $\alpha, \beta$, and $\gamma$ can be evaluated from annealing experiments. Figure 1 shows the experimental results given by Hull et $a l .{ }^{6}$ for the growth and growth annealing of $x=0.25$ structures at a growth rate of $0.3 \mathrm{~nm} \mathrm{~s}^{-1}$. Experimental data were given for $550{ }^{\circ} \mathrm{C}$ growth and for $550{ }^{\circ} \mathrm{C}$ growth +10 min $800^{\circ} \mathrm{C}$ in situ annealing in a molecular beam epitaxy (MBE) chamber for the average measured mismatch dislocation spacing $p$ versus epitaxial layer thickness $h$. After 10 min annealing at $800^{\circ} \mathrm{C}$ the materials system arrived at the equilibrium state. The equilibrium relaxed plastic strain can be obtained from Eqs. (6) and (7) by setting $\tau_{\mathrm{exc}}-\tau_{R}=0$,

$$
\epsilon_{p}=\epsilon_{0}-\frac{K_{0}}{h}-H\left(\epsilon_{p}\right)
$$

For the $\mathrm{Ge}_{x} \mathrm{Si}_{1-x} / \mathrm{Si}(100)$ material system, $\nu=0.22$ and $b=0.384 \mathrm{~nm}$. In this article we choose $h_{0}=35 \mathrm{~nm}$, then $K_{0}$ $=0.123 \mathrm{~nm}$ which gives a very good prediction of the critical thickness as shown in Fig. 4. From three experimental data points corresponding to $550^{\circ} \mathrm{C}$ growth followed by a $10 \mathrm{~min}$ anneal in Fig. 1, one can easily obtain parameters $\alpha, \beta$, and $\gamma$. We have $\alpha=0.0159, \beta=0.167$, and $\gamma=5.0$. In order to obtain parameters $v_{0}$ and $\xi_{0}$, let us look at the experimental results of Hull et al. ${ }^{6}$ for 4 min thermal annealing of a $35 \mathrm{~nm}$ thick $\mathrm{Ge}_{x} \mathrm{Si}_{1-x} / \mathrm{Si}(100)$ layer with $x=0.25$ after growth at each temperature. Figure 2(a) shows the average mismatch dislocation spacing $p$ versus growth-annealing temperature $T$ and Fig. 2(b) shows the threading dislocation density $\rho$ versus growth-annealing temperature $T$. Only using two experimental data points at a temperature of $550{ }^{\circ} \mathrm{C}$ in Figs 2(a) and $2(\mathrm{~b})$, respectively, one can easily get parameters $v_{0}$ and $\xi_{0}$. They are $v_{0}=0.16 \times 10^{14} \mathrm{~nm} \mathrm{~s}^{-1}$ and $\xi_{0}=0.27$ $\times 10^{10} \mathrm{~nm}^{-2} \mathrm{~s}^{-1}$. All together only five experimental data points are used for determining the material parameters $\alpha, \beta$, $\gamma, v_{0}$, and $\xi_{0}$.

\section{RESULTS AND DISCUSSIONS}

Now all material parameters are known. One can simulate the kinetic process for the $\mathrm{Ge}_{x} \mathrm{Si}_{1-x} / \mathrm{Si}(100)$ material system. The present model's results for growth and growth annealing of the $x=0.25$ structure are also shown in Fig. 1 . Three experimental data points for $550{ }^{\circ} \mathrm{C}$ growth are within a factor of $\sim 2$ of the theoretical curve and the other three data points for $550{ }^{\circ} \mathrm{C}$ growth $+800{ }^{\circ} \mathrm{C}$ annealing for $10 \mathrm{~min}$ are a little lower than the theoretical curve. It means that when the thickness is less than $50 \mathrm{~nm}$ the simulated evolution process does not arrive at a real equilibrium state yet, and thereby a longer annealing time is needed for the simulated system to arrive at the real equilibrium state.

A comparison of the experimental results and the present simulation results for growth and 4 min thermal annealing of a $35 \mathrm{~nm}$ thick $\mathrm{Ge}_{x} \mathrm{Si}_{1-x} / \mathrm{Si}(100)$ layer with $x=0.25$ at different temperatures is also shown in Fig. 2. Figure 2(a) shows the final mismatch dislocation spacing $p$ at different temperatures. As the growth-annealing temperature $T$ increases, the final relaxed plastic strain $\epsilon_{p}$ increases; then according to Eq. (8), the final average mismatch dislocation spacing $p$ decreases. On the other hand, the final threading dislocation density $\rho$ increases rapidly as the temperature increases. The final relaxed plastic strain $\epsilon_{p}$ versus the growthannealing temperature $T$ is shown in Fig. 2(c). From Figs. 2(a)-2(c) one can see that the present theoretical results agree well with the experimental data.

Figure 3 shows a comparison of experimental results and the present simulation results for final elastic strain $\left(\epsilon_{0}\right.$ $-\epsilon_{p}$ ) versus initial mismatch strain $\epsilon_{0}$ following the growth of $\mathrm{Ge}_{x} \mathrm{Si}_{1-x} / \mathrm{Si}(100)$ at a temperature of $550{ }^{\circ} \mathrm{C}$. The experi- 

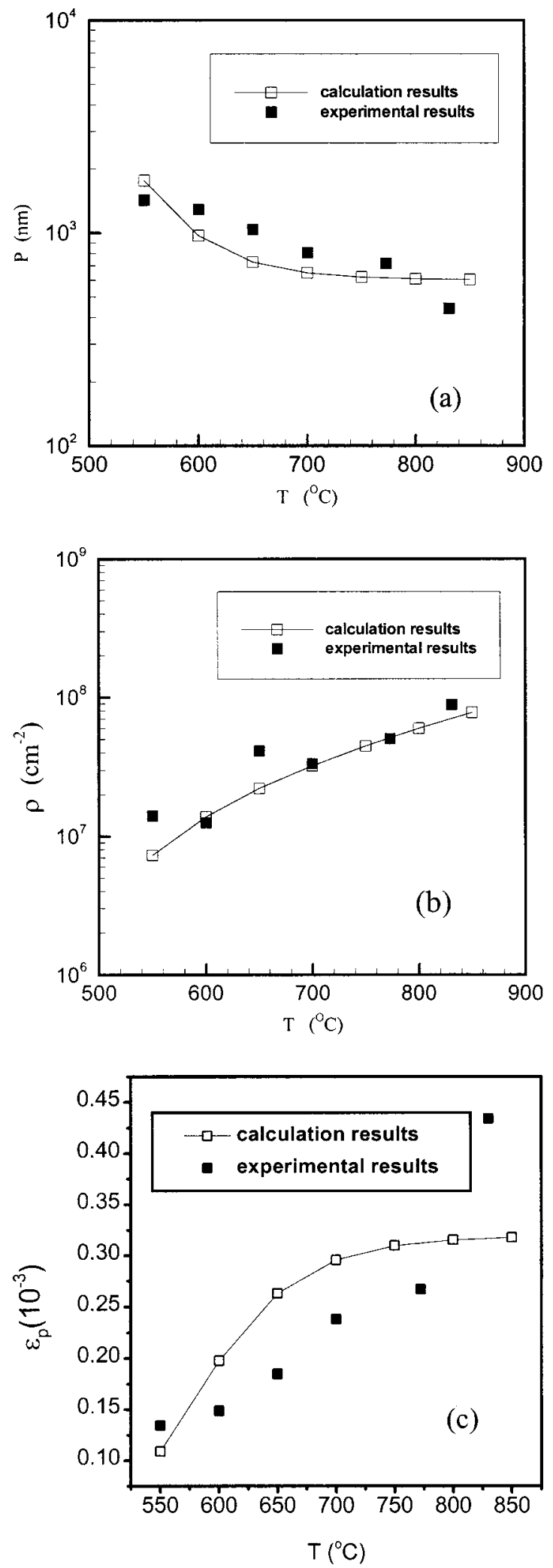

FIG. 2. Comparison of experiment and present modeling for growth annealing of a $35 \mathrm{~nm}$ thick $x=0.25 \mathrm{Ge}_{x} \mathrm{Si}_{1-x} / \mathrm{Si}(100)$ structure. The experimental data, shown by closed points, were given by Hull et al. (see Ref. 6) for growth and a 4 min annealing at each temperature. The growth rate is 0.3 $\mathrm{nm} \mathrm{s}^{-1}$. (a) Average mismatch dislocation spacing $p$ vs growth-annealing temperature $T$, (b) threading dislocation density $\rho$ vs the temperature $T$, (c) relaxed plastic strain $\epsilon_{p}$ vs the temperature $T$. All open points are the present calculation results.

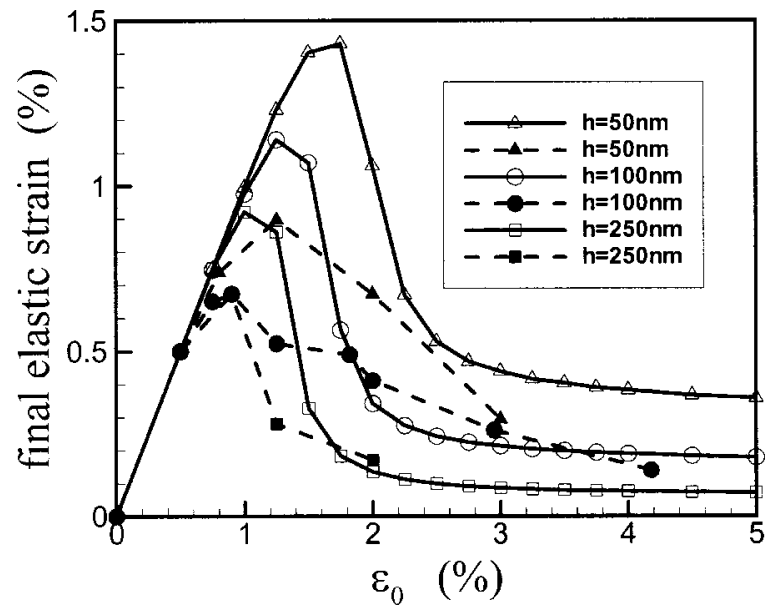

FIG. 3. Final elastic strain vs initial mismatch strain following growth of a $\mathrm{Ge}_{x} \mathrm{Si}_{1-x} / \mathrm{Si}(100)$ material system at a temperature of $550{ }^{\circ} \mathrm{C}$. The experimental data, shown by closed points, were given by Bean et al. (see Ref. 11) at a growth rate of $0.5 \mathrm{~nm} \mathrm{~s}^{-1}$. All open points are the present calculation results.

mental data measured by $\mathrm{x}$ ray were those given by Bean et $a l .{ }^{11}$ Critical thickness theory states that the final elastic strain is only dependent on the epitaxial layer thickness $h$ no matter what the value of $\epsilon_{0}$. This is in total contrast with the experimental observations. The experimental observations given by Bean et al. ${ }^{11}$ clearly show that the final elastic strains do vary remarkably with the initial mismatch. The present simulation captures the trend of this phenomenon well.

A comparison of the experimental measurements and the predictions of different theories for the critical thickness in the $\mathrm{Ge}_{x} \mathrm{Si}_{1-x} / \mathrm{Si}(100)$ structure is shown in Fig. 4. The dashed line and the solid line are the predictions of the Matthews-Blakeslee model for two different values of core energy parameters: $\alpha=1$ and 4, respectively. The open tri-

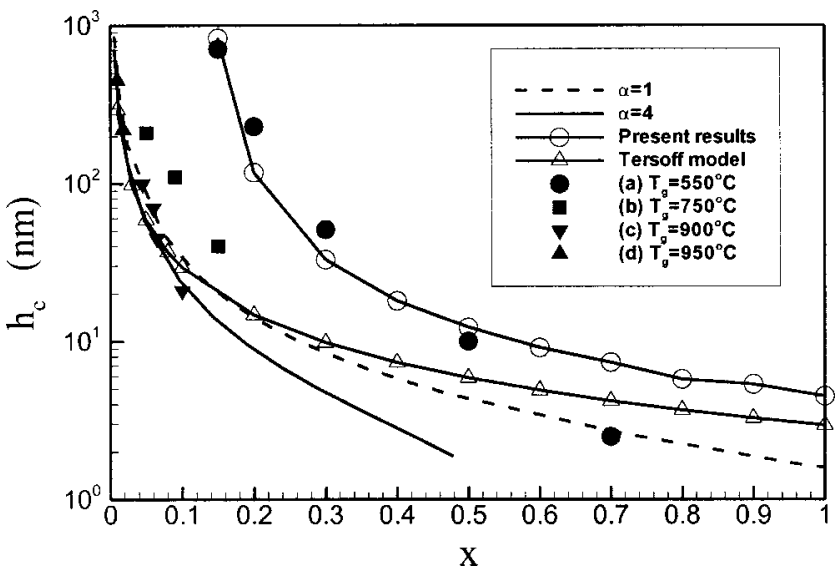

FIG. 4. Predictions of different models for critical thickness, $h_{c}$ in the $\mathrm{Ge}_{x} \mathrm{Si}_{1-x} / \mathrm{Si}(100)$ structure and experimental measurements of $h_{c}$ for different growth temperatures $T_{g}$ from the work of (a) Bean et al. (see Ref. 11) $\left(T_{g}=550{ }^{\circ} \mathrm{C}\right)$, (b) Kasper et al. (see Ref. 15) $\left(T_{g}=750{ }^{\circ} \mathrm{C}\right)$, (c) Green et al. (see Ref. 12) $\left(T_{g}=900^{\circ} \mathrm{C}\right)$, and (d) Houghton et al. (see Ref. 16) $\left(T_{g}=950^{\circ} \mathrm{C}\right)$. 
angles are the predictions of Tersoff's model with $K_{0}$ $=0.123 \mathrm{~nm}$. These predictions agree well with the measurements by Green et al. ${ }^{12}$ and by Houghton ${ }^{7}$ at high temperature. But the measurements given by Bean et al. ${ }^{11}\left(T_{g}\right.$ $=550{ }^{\circ} \mathrm{C}$ ) are significantly higher than the predictions of the Matthews and Blakeslee model. ${ }^{13}$ It means that with low temperature growth there is a kinetic barrier to dislocation nucleation and propagation. As pointed out by Fritz ${ }^{14}$ and by Hull et al. ${ }^{6}$ the experimental measurements of the critical thickness will be a sensitive function of the experiment techniques used to measure strain relaxation. If we choose a thickness as the effective critical thickness at which the plastic strain relaxation is of $10^{-4}$, then we can use the evolution equations to predict the effective critical thickness. The small open circles are the predictions of present evolution equations on the effective critical thickness for growth at a temperature of $550{ }^{\circ} \mathrm{C}$. The predictions show good agreement with the experiment data (closed circles).

\section{ACKNOWLEDGMENTS}

The authors would like to thank Dr. P. M. Moran for reviewing the manuscript and one of the authors (T.C.W.) wishes to acknowledge the financial support by The National University of Singapore, where he spent his sabbatical leave.

${ }^{1}$ W. K. Liu and M. B. Santos, Thin Films: Heteroepitaxial Systems (World Scientific, London, 1999).

${ }^{2}$ J. Y. Tsao, Materials Fundamentals of Molecular Bean Epitaxy (Academic, Boston, 1993)

${ }^{3}$ E. A. Fitzgerald, A. Y. Kim, M. T. Currie, T. A. Langdo, G. Taraschi, and M. T. Bulsara, Mater. Sci. Eng., B 67, 53 (1999).

${ }^{4}$ A. Y. Kim, W. S. McCullough, and E. A. Fitzgerald, J. Vac. Sci. Technol. B 17, 1485 (1999).

${ }^{5}$ B. W. Dodson and J. Y. Tsao, Appl. Phys. Lett. 51, 1325 (1987).

${ }^{6}$ R. Hull, J. C. Bean, and C. Buescher, J. Appl. Phys. 66, 5837 (1989).

${ }^{7}$ D. C. Houghton, J. Appl. Phys. 70, 2136 (1991).

${ }^{8}$ T. J. Gosling, S. C. Jian, and A. H. Harker, Phys. Status Solidi A 146, 713 (1994).

${ }^{9}$ L. B. Freund, Advances of Applied Mechanics (Academic, San Diego, 1994), Vol. 30, p. 1

${ }^{10}$ J. Tersoff, Appl. Phys. Lett. 62, 693 (1993).

${ }^{11}$ J. C. Bean, L. G. Feldman, A. T. Fiory, S. Nakahara, and I. K. Robinson, J. Vac. Sci. Technol. A 2, 436 (1984).

${ }^{12}$ M. L. Green, B. E. Weir, D. Brasen, Y. F. Hsih, G. Higashi, A. Feygenson, L. G. Feldman, and R. H. Headrick, J. Appl. Phys. 69, 745 (1991).

${ }^{13}$ J. W. Matthews and A. E. Blakeslee, J. Cryst. Growth 27, 118 (1974).

${ }^{14}$ I. J. Fritz, Appl. Phys. Lett. 51, 1080 (1987).

${ }^{15}$ E. Kasper, H.-J. Herzog, and H. Kibbel, Appl. Phys. 8, 199 (1975).

${ }^{16}$ D. C. Houghton, C. J. Gibings, C. G. Tuppen, M. H. Lyons, and M. A. G. Halliwell, Appl. Phys. Lett. 56, 460 (1990). 